\title{
Relativistic Effects on X-ray Emissions from Accretion Disks around Black Holes
}

\author{
Xiaoling Zhang*, Shuang Nan Zhang* and Yangsen Yao* \\ ${ }^{*}$ University of Alabama in Huntsville and National Space Science and Technology Center, \\ Physics Department, Huntsville, AL 35899, USA
}

\begin{abstract}
Special and general relativistic effects on the X-ray emissions, especially the continuum spectra, from the accretion disks around black holes are investigated using the ray-tracing method. Because both the special and general relativistic effects are more important at distances closer to the black hole, the relativistic modifications to the emitted X-ray spectra are more significant at higher energies. In a simple accretion disk precession model, the relativistic effects can account for the energy dependence of the QPO amplitude and phase-lags observed in several black hole binaries. The dramatic QPO phase-lag transitions only show up when the central black hole is spinning rapidly. The narrow distribution of the observed system inclination angles of black hole binaries may be due to the selection effect caused by the relativistic effects around black holes.
\end{abstract}

\section{INTRODUCTION}

Many black hole binaries have been known and studied in recent years. Because of the strong gravitational field near the black hole, the radiation from the inner portion of the accretion disk experiences strong relativistic modifications before escaping from the system. In order to infer the physical parameters of the accretion disk and the black hole from the observed X-ray radiation, it is necessary to consider the relativistic effects (e.g., Zhang, Cui \& Chen 1997; Cui, Zhang \& Chen 1998). The exact modifications depend strongly on the angular momentum of the black hole and the inclination angle of the disk, as well as the black hole mass and the accretion rate.

Analytical calculations of the relativistic modifications can only be done in a few very simple cases. For most applications, numerical methods must be adopted. Here we use the ray-tracing method by Fanton et al. (1997), assuming that the disk is a thin Keplerian disk. The ray-tracing method takes all special and general relativistic effects into account.

In this paper we calculate the relativistically modified X-ray spectra from black hole $\mathrm{X}$-ray binaries with different angular momenta and disk inclination angles. In particular we investigate the X-ray light curve modulations if the accretion disk is assumed to precess. Our calculations reproduce the observed light curve modulation rms (rootmean-squares) and the peculiar phase-lags around QPO peaks from the microquasar GRS1915+105, if the black hole in GRS1915+105 is spinning rapidly. Our results thus suggest that the observed light curve modulations and phase-lags are manifestation of the strong relativistic effects around a rapidly spinning black hole. We also propose that the observed system inclination angles around 60 to 70 degrees for all black hole binaries may be due to the selection effect caused by the relativistic effects around black holes. 


\section{MODELS FOR X-RAY SPECTRA AND FLUX MODULATIONS}

We follow the widely established model for the X-ray spectra from black hole X-ray binaries, i.e., the two component model consisting of a soft, blackbody-like component and a hard, power-law like component. The soft component is usually approximated by the diskbb model in the XSPEC package. Here we use the radial temperature profile derived in the Kerr metric for the soft component.

For the hard component, currently there is no widely accepted physical model yet. For simplicity, we assume that the hard component is also produced from very close to the accretion disk. We further assume that the local hard component is of a power-law shape with a low energy cutoff. The local power-law photon index is assumed to be $\alpha=$ $2.2+\left(r / r_{g}\right) / 40$ in order to mimic the observed power-law shape. The low energy turnover is the same as that in the Comptonization model (Sunyaev and Titarchuk 1980) i.e., implying that the power-law is produced via (thermal or non-thermal) Comptonization and the seed photons are the local blackbody emission from the disk. The emissivity of the hard and soft component is assumed to be the same, i.e., the gravitational energy release to X-ray radiation is shared equally in the soft and hard component.

We consider all relativistic effects when calculating the X-ray spectra at infinity. First, the rapid Keplerian motion of the disk causes both the Doppler frequency shift and Doppler boosting. Secondly, the strong gravitational field introduces the gravitational redshift and focusing. These effects can be expressed with the transfer function (Cunningham, 1975), calculated here with the ray-tracing method of Fanton et al. (1997).

\section{RESULTS OF RAY-TRACING CALCULATIONS}

In Fig. 1, we show the calculated X-ray spectra at infinity, for different black hole angular momenta and disk inclination angles. We fixed the black hole mass and mass accretion rate, such that the peak temperature for the extremal Kerr black hole is $2.1 \mathrm{keV}$; for all other black hole angular momenta, the corresponding peak temperatures are lower accordingly. These spectra may be fitted using the XSPEC package with the RXTE PCA response and diskbb+powerlaw model, with reasonable parameters and residuals, demonstrating that our spectral model can indeed reproduce the observed X-ray spectra from black hole X-ray binaries.

In Fig. 2, we show the observed flux in different energy bands as a function of the disk inclination angle. It is clear that significant deviations from the cosine law exist for a rapidly spinning black hole, especially at higher energies. This demonstrates clearly the necessity of taking into account relativistic effects if the black hole is spinning.

In a simple disk precession model, we may assume that the whole inner disk region precesses at a certain period, which is determined by the exact physical mechanism and mode of the disk precession. The observed X-ray flux will then display periodic oscillations. As an example, we assume that the disk between the last stable orbit and $200 r_{g}$ undergoes stable precession with inclination angles between 60 and 80 degrees. No other source of flux modulation is assumed to exist. In Fig. 3, the observed light curves in different energy bands are plotted for different black hole angular momenta. It is striking that the low energy and high energy light curves are opposite in phase for a rapidly spinning black hole. 


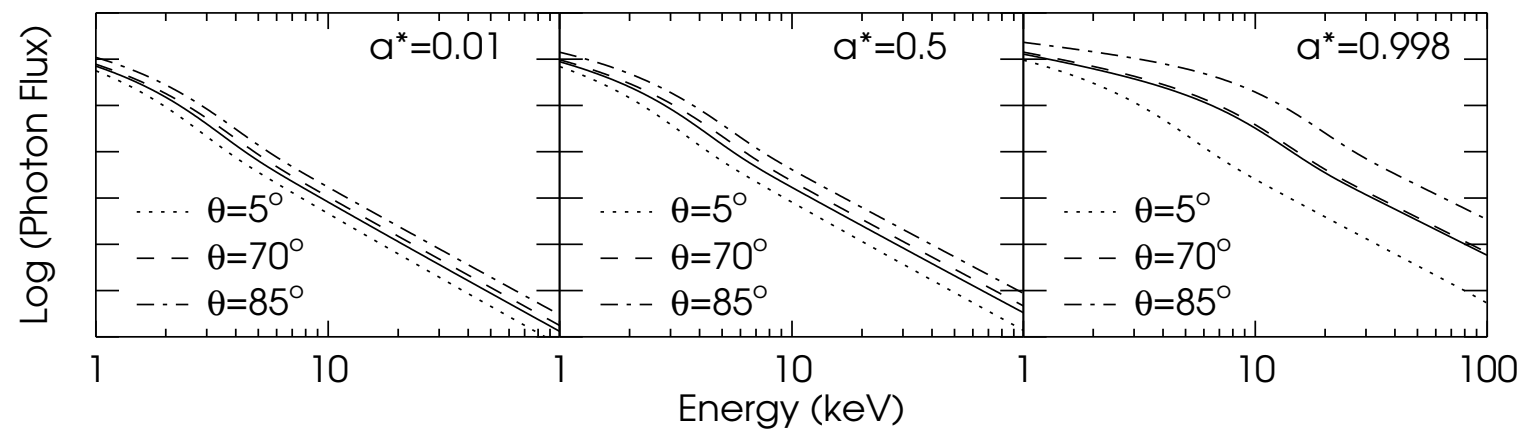

FIGURE 1. Accretion disk spectra around black holes with different angular momenta and viewed with different inclination angles from infinity. The solid lines are the local spectra and the dotted lines are the spectra observed at infinity, after modifications by special and general relativistic effects. For a rapidly spinning black hole system, the observed spectrum at infinity above $10 \mathrm{keV}$ may deviate from the local spectrum significantly.

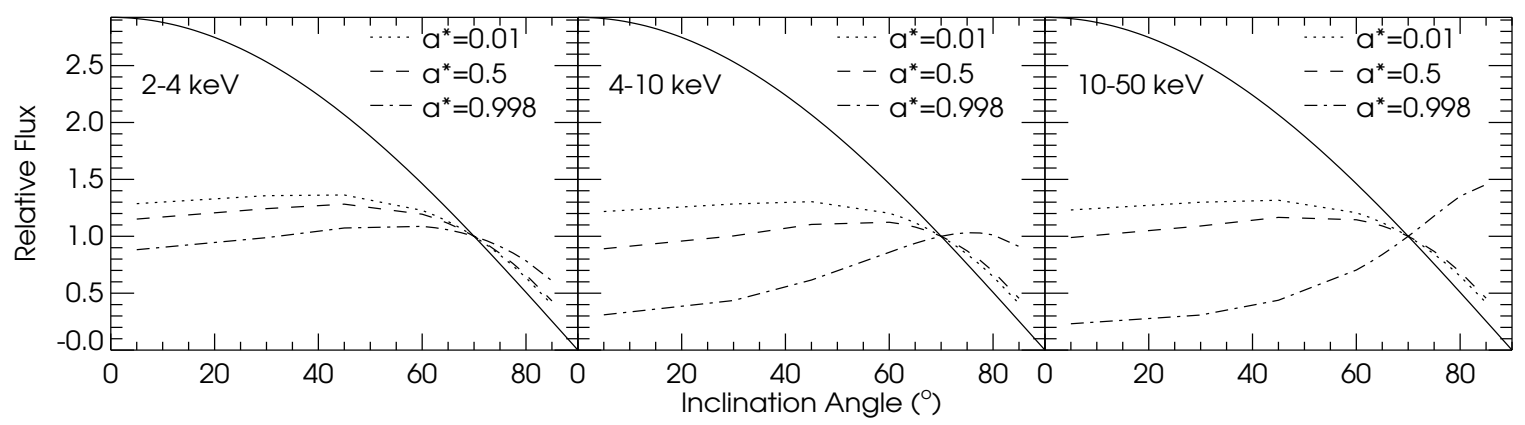

FIGURE 2. Flux at infinity in different energy bands as a function of the inclination angle after the relativistic modifications, as shown by the dotted lines; the solid lines are for the cosine law, appropriate when relativistic effects are negligible. The deviations from the cosine law are very significant for a rapidly spinning black hole, especially in high energy bands. This indicates that the observed light curve modulations in different energy band will not be in phase when the disk is forced to precess.

In Fig. 4, we show two sample power density spectra and the phase-lag spectra between them for different black hole angular momenta, with or without any white noise. Relatively strong harmonics of the precession frequency are observed. When $a^{*}=0.5$ no phase-lag is present, because the low and high energy light curves are in phase. However, when $a^{*}=0.998$, the low and high energy light curves are always opposite in phase. However, in the existence of a weak white noise, only sharp phase-lag transitions are observed around the peaks of the power density spectra.

Finally in Fig. 5, we show the rms of the light curve modulations as a function of energy for different black hole angular momenta. For non- and slowly spinning black holes, the light curve modulation rms does not change significantly as a function of energy. However, for the case of an extremal Kerr black hole, the light curve modulation depends strongly on the photon energy.

In summary, when the black hole is spinning rapidly, sharp phase-lag transitions and strong energy dependence of the X-ray light curves are expected. Therefore the X-ray light curves may be used for probing the strong relativistic effects near black holes. 


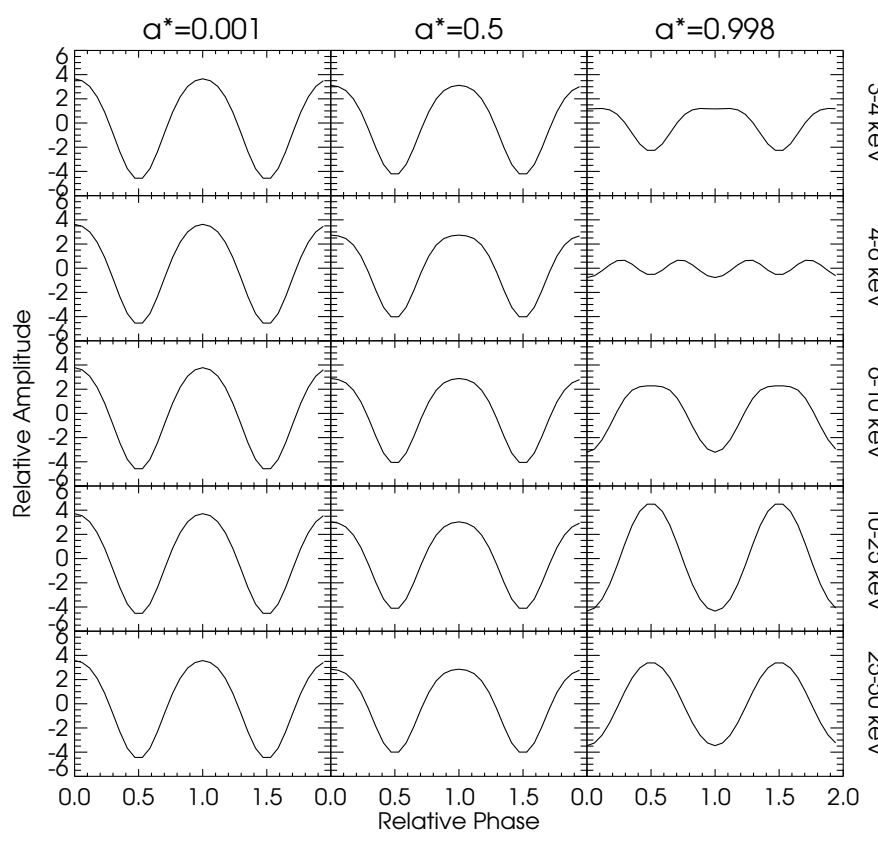

FIGURE 3. Observed light curves in different energy bands at infinity. The whole accretion disk responsible for X-ray emission is assumed to precess at a certain frequency and the precession is assumed to be the only source of the observed light curve modulation. Note that for a non- and slowly spinning black hole, the low and high energy light curves are in phase. However when $a^{*}=0.998$, the light curves in different energy bands are very different; low energy and high energy light curves are in fact opposite in phase.
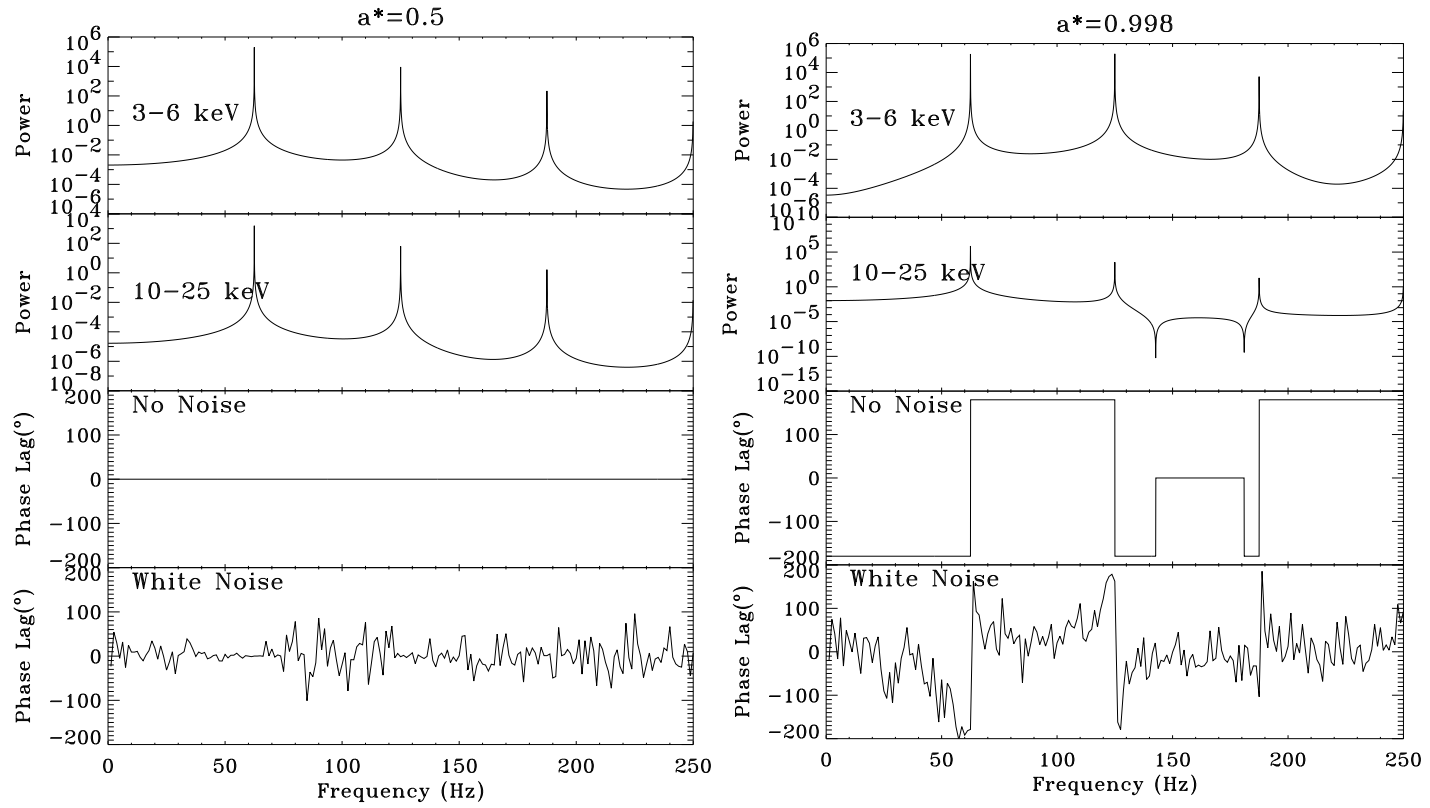

FIGURE 4. Sample power density and phase lag spectra, for two light curves in 3-6 keV and 10-25 keV bands. The white noise is about $0.2 \%$ of the signals. For the extremal Kerr black hole $\left(a^{*}=0.998\right)$, sharp phase-lag changes are expected around the peaks of the power density spectrum. The sharp phase-lag changes are purely due to the relativistic effects around a rapidly spinning black hole.

\section{DISCUSSIONS}

The most striking result of this investigation is the naturally produced sharp phaselag transitions around the peaks in the power-density spectra. Regardless the details of 


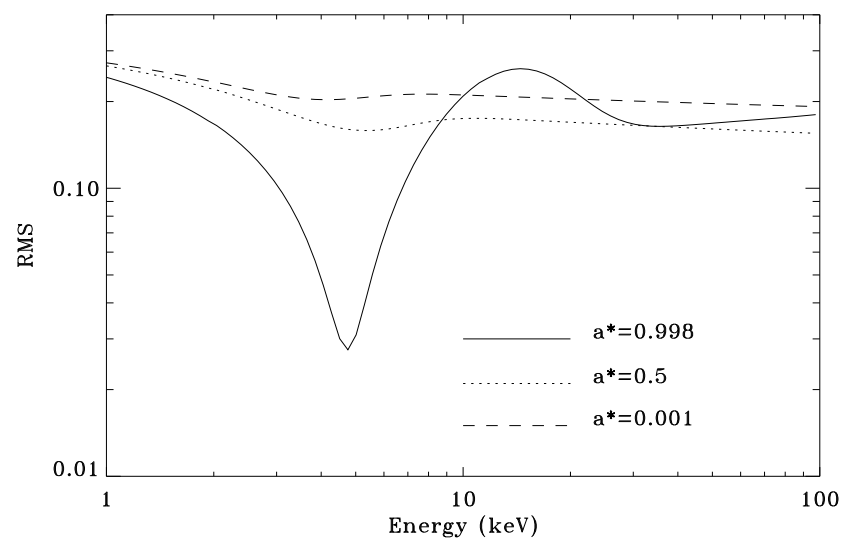

FIGURE 5. The rms (root-meansquares) of the light curve modulations, as a function of energy. For non- and slowly spinning black holes, the light curve modulation rms does not change significantly as a function of energy. However, for the case of an extremal Kerr black hole, the light curve modulation depends strongly on the photon energy.

our calculations, this feature is robust and relies essentially on only three assumptions: higher energy photons are produced at distances closer to the black hole, the periodic flux modulations are mainly due to the accretion disk precession, and the black hole is spinning rapidly. This phenomenon has been observed in GRS1915+105 (Cui, 1999; Lin et al. , 2000). The increasing trend of the light curve rms for an extremal Kerr black hole in the middle energy range, as shown in Fig. 5, has also been detected in GRS1915+105 (e.g., Cui, 1999). Therefore our results confirm that the black hole in GRS1915+105 is spinning rapidly (Zhang, Cui \& Chen 1997; Cui, Zhang \& Chen 1998). From Fig. 5, we predict that the rms decreases at energies above about 20-30 keV for extremal Kerr black hole binaries.

Finally our calculations may shed light on the outstanding puzzle that almost all observed X-ray black hole binaries have inclination angles around $60^{\circ}-70^{\circ}$ (Zhang et al. 1997). The combined effects of higher space density for high inclination angle systems and the relative flux of these high inclination angle systems (see Fig. 2) favor detection of binary systems at inclination angles around $60^{\circ}-70^{\circ}$. Thus it is likely that the inclination angle distribution of known black hole binaries is simply a selection effect favoring the brightest X-ray sources, manifesting the strong relativistic effects around black holes.

Acknowledgments: We thank Dr. Fanton for providing us with the ray-tracing code. We have also benefited from discussions with Drs. Lev Titarchuk, Wei Cui, Yuxin Feng and Dingxiong Wang. This work was supported in part by NASA MSFC under contract NCC8-200 and by NASA LTSA Program under grants NAG5-7927 and NAG5-8523.

\section{REFERENCES}

1. Cui, Wei, Zhang, S.N., Chen, Wan, 1998, ApJ, 492, L53

2. Cui, Wei, 1999, ApJ, 524, L59

3. Cunningham, C. T., 1975, ApJ, 202, 788

4. Fanton, C., et al. , 1997, PASJ, 49, 159

5. Lin, D., Smith, I.A., Liang, E.P., and Bottcher, M., 2000, ApJ, 543, L141

6. Makishima, K. et al. , 1986, ApJ, 308, 635

7. Sunyaev, R. A., \& Titarchuk, L. G., 1980, A\&A, 86, 121

8. Zhang, S. N., Cui, Wei, and Chen, Wan, 1997, ApJ, L155

9. Zhang, S. N.,et al. , 1997, Invited Review of the 4th Compton Symposium Proceedings, AIP Conference Series, 410, 141, eds Charles D. Dermer, Mark S. Strickman, James D. Kurfess. 